\title{
La comunicación integrada y la narrativa transmedia
}

Integrated communication and transmedia storytelling

Ana Tázia Patricio De Melo Cardoso, Universidade Potiguar -

Patricia Gallo, Universidade Potiguar -

Resumen

En medio a tanta información, conseguir la atención de la audiencia no es una simple tarea para las empresas, ya que la audiencia viene alterando sus prácticas de consumo, de comunicación y de sociabilidad, especialmente por la posibilidad de integración de los espacios físico y virtual y de la multi-direccionalidad de los mensajes.

Es en este escenario de proliferación de medios, de sobrecarga informacional, de atención flexible de la cultura participativa y de mercado globalizado que la comunicación integrada se alía a la narrativa transmedia como estrategia competitiva y como experiencia multimodal, para generar una relación comunicativa contínua y duradera entre marca y consumidor, potencializando la efectividad de la compra.

Palabras clave

Comunicación, narrativa, transmedia.

\section{Abstract}

In the midst of so much information, getting the attention of the audience is not a simple task for companies, as the audience is altering its consumption, communication and sociability practices, especially due to the possibility of integrating physical and virtual spaces and the multi-directionality of messages.

It is in this scenario of media proliferation, information overload, flexible attention to participatory culture and a globalised market that integrated communication is allied to transmedia narrative as a competitive strategy and as a multimodal experience, to generate a continuous and lasting communicative relationship between brand and consumer, enhancing the effectiveness of the purchase.

\section{Palabras clave}

Communication, narrative, transmedia

\section{Sumario}

1. Comunicación Integrada. 2. La Narrativa Transmedia. 3. Una Estrategia y Experiencia Comunicacional. 4. Consideraciones finales. 5. Referencias Bibliográficas. 


\section{Comunicación Integrada}

Cada vez más se percibe que la integración de las acciones de comunicación es la mejor forma de atingir la gran diversidad de los públicos organizacionales.

La definición del enfoque, su posicionamiento de mercado y cliente-objetivo son aspectos cruciales para que se pueda consolidar una marca, por lo tanto, su desarrollo y su concepción necesitan estar centrados en una estrategia empresarial. De esta forma se busca el reconocimiento del consumidor y, para que esto en realidad ocurra, las herramientas de comunicación son fundamentales al generar real valor a la marca.

Para que una empresa alcance sus objetivos de comunicación es necesario dedicar mucha atención a su comunicación organizacional. De acuerdo con la estructura (Figura 1) propuesta por Kunsch (2003) para la comunicación organizacional, se apunta los tipos de comunicación que integran el compuesto comunicacional: institucional, mercadológica, interna y administrativa.

Figura 1: Cronograma de comunicación organizacional integrada

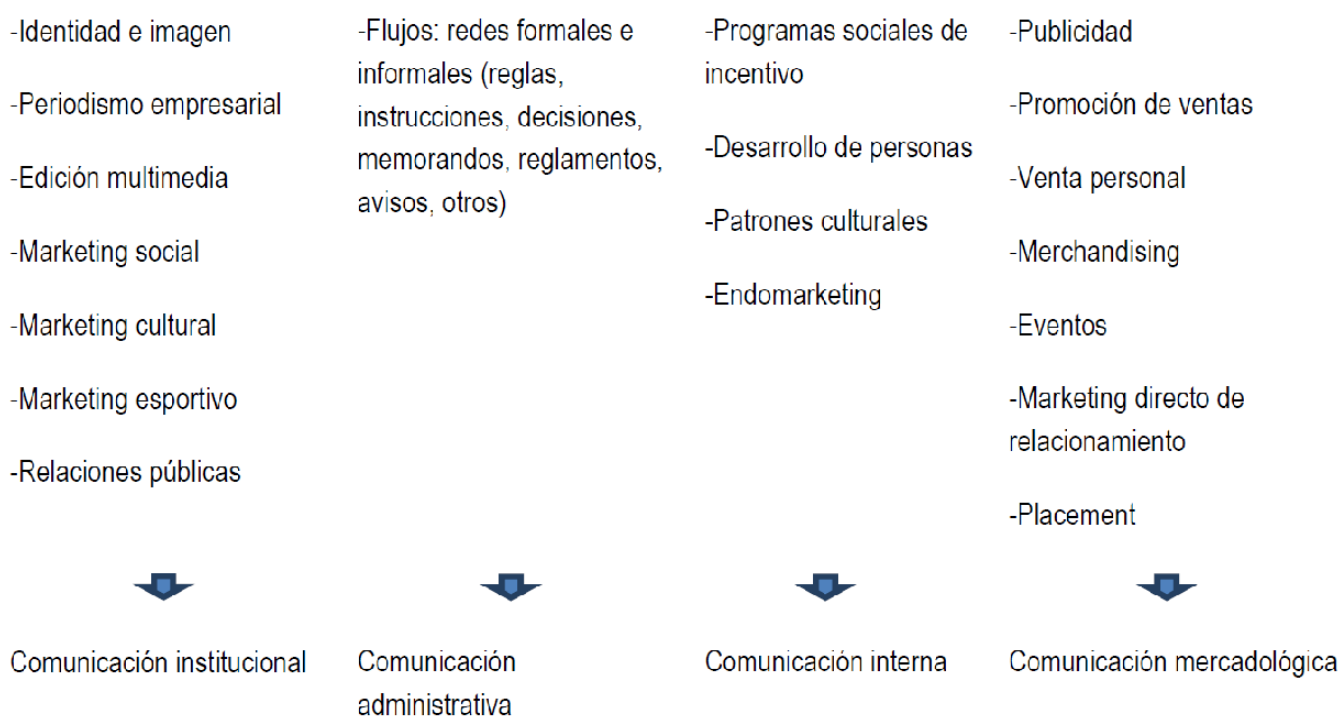

Fuente: KUNSCH (2003)

Bajo esta perspectiva podemos entender la comunicación organizacional como un fenómeno intrínseco a las agrupaciones de personas de una organización o que a ella están vinculadas, tal como destaca Kunsch (2003). Cabe a la gestión de comunicación integrada de la empresa sacar el máximo partido de cada una de las herramientas presentadas y buscar integrarlas de manera a obtener sinergia en el esfuerzo de comunicación.

Es importante que haya una acción planeada para coordinar todo el mix, de ahí la necesidad de integración desde la comunicación interna, con objetivos bien definidos, para viabilizar toda la interacción posible entre organización y empleados, incluso al utilizar herramientas de comunicación institucional e incluso de la comunicación mercadológica. También uno de los pilares de la comunicación integrada, la comunicación mercadológica, visa promover intercambios y/o ventas, interactuando con el mercado - de modo que aporte resultados a la organización, a los gestores y colaboradores. Al caracterizarse por la relación publica de la organización con la sociedad, a través de cuestiones políticas y sociales, la comunicación institucional viabiliza la construcción de la imagen de la organización a través de una interacción directa entre la empresa y su público.

Los sectores de comunicación de las empresas desempeñan un importante papel en la creación del valor de la marca y también en la formación de una sólida fidelidad a ella. Al adoptar un abordaje integrado en sus actividades de comunicación, no podemos ser apenas seguidores de los formatos ya existentes pero sí desbravadores de nuevos medios y formatos para que la comunicación sea siempre vista por la sociedad como algo creativo, diferenciado e estimulante. En este sentido, visualizamos que la comunicación integrada aliada a la narrativa transmedia favorece este proceso. 


\section{La Narrativa Transmedia}

La palabra Transmedia significa, por si misma a través de medios y se refiere a cualquier combinación de las relaciones que pueden existir entre diversos textos (digitales y analógicos) que constituya una experiencia mediática en entretenimiento contemporáneo (Jerkins et al.,

2013, traducción libre de las autoras). O sea, Transmedia es el movimiento del contenido o del mensaje en las diversas plataformas de medios. Ella tiene su actuación y aplicación por categorías, definidas de acuerdo con los objetivos que se intenta alcanzar, de acuerdo con el área de actuación. No obstante, todas las categorías traen como principio la participación de la audiencia en un universo a ser e explorado a través de los medios.

Las categorías reconocidas por la literatura científica internacional son: Transmedia Storytelling (narrativa transmedia), Transmedia Branding, Transmedia Play, Transmedia Learning e Transmedia Actvism. En el presente texto, hacemos referencia apenas a la narrativa transmedia, pues ella viene siendo la categoría más conocida y estudiada, incluso porque constituye la base de las demás categorías.

Según Jenkins (2009, p. 49), la narrativa transmedia "se refiere a una nueva estética que ha surgido en respuesta a la convergencia de los medios - una estética que hace nuevas exigencias a los consumidores y depende de la participación activa de comunidades de conocimiento. Al mencionar una nueva estética, Jenkins focaliza el universo a ser explorado como una fruición estética participativa.

Aún según el autor, este tipo de narrativa "se desenvuelve a través de múltiples plataformas de medios, con cada nuevo texto contribuyendo de manera distinta y valiosa para el todo" (2009, p.

138).

En resumen, en un proceso de narrativa transmedia, los elementos que la componen distribuidos sistemática y estratégicamente a través de medios, criando una experiencia unificada y coordinada en entretenimiento contemporáneo. La participación del público ocurre por la exploración del universo creado. 0 sea, los individuos necesitan asumir el papel de cazadores, siguiendo el flujo y las partes de contenido o mensajes por diferentes medios, comparando y compartiendo sus encuentros y descubiertas a través de las herramientas online con otros individuos. Así, juntos, llegan en nuevas descubiertas. Es este movimiento de exploración colectiva que convierte la experiencia de interacción con la marca/empresa en algo más envolvente y rico.

La figura 2 presenta un universo a ser explorado, considerando la comunicación integrada y la narrativa transmedia como estrategia de gestión comunicacional de una empresa/marca.

Figura 2: La narrativa transmedia y comunicación integrada

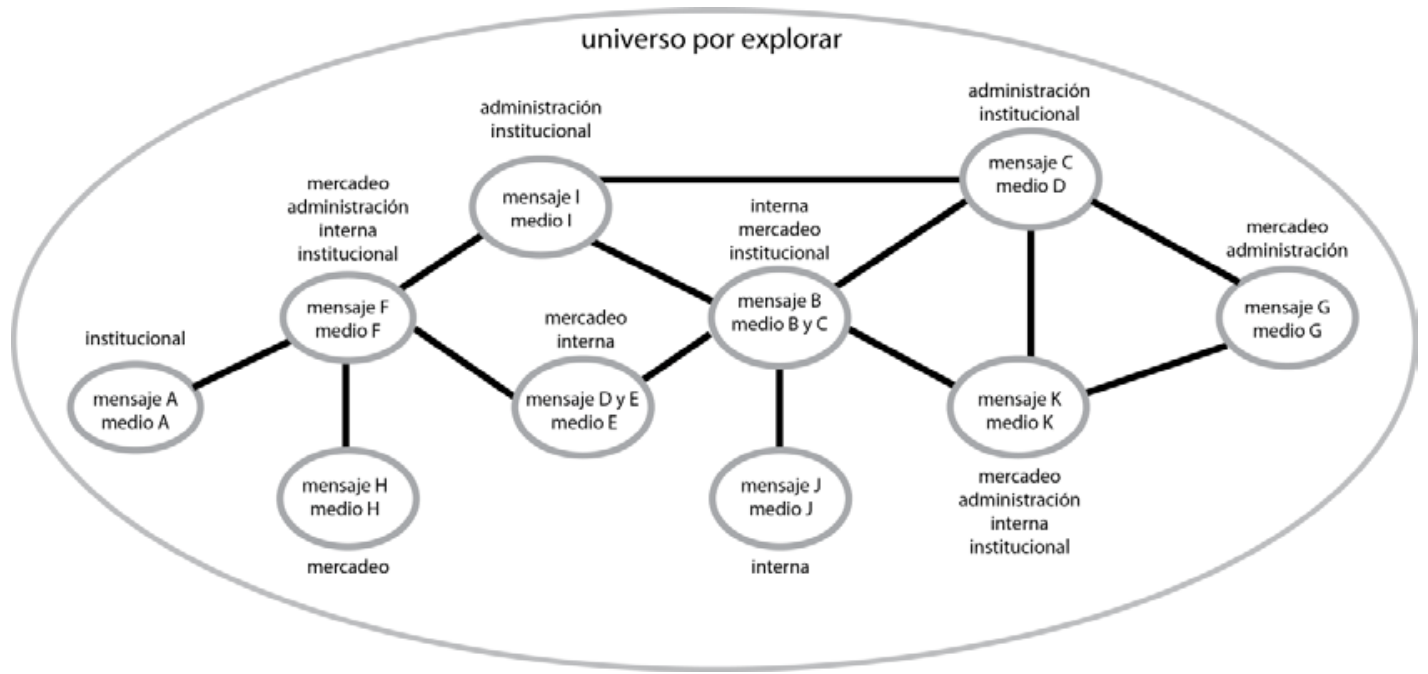

Fuente: de las autoras

Jenkins enfatiza que cada medio necesita hacer el que hace de mejor, considerando sus potencialidades y los accesos de entrada en el universo deben ser autónomos, o sea, sin que haya una linealidad obligatoria de exploración, el público transita espontáneamente por la narrativa, según sus intereses y necesidades. Con esto, el entendimiento de cada individuo sobre el universo será único.

El entretenimiento fue el primer segmento que ha visualizado las potencialidades socioculturales y mercadológicas de la narrativa transmedia. Entretanto, con las adaptaciones necesarias, pues los contextos de uso en el entretenimiento y en otras áreas y segmentos son distintos, ese tipo de narrativa viene despertando el interés de profesionales, especialmente por dar oportunidad a un proceso 
interactivo envolvente y duradero que, aliado a los objetivos bien definidos, viene trayendo los resultados comunicacionales y/o mercadológicos esperados.

Un ejemplo de actuación con resultados positivos es la utilización de la narrativa transmedia en la gestión de las marcas y en campañas publicitarias y acciones de marketing, insiriendo la trayectoria de personajes primarios y secundarios, con sus historias de vida llenas de objetivos, conflictos y transformaciones.

En una estructura de narrativa, es en el problema anunciado (conflicto) que el envolvimiento puede ocurrir. 0 sea, los individuos dispuestos a interaccionar con la narrativa, van en busca del desfecho. Esa busca pasa por variados medios, ya que las partes de la narrativa están difundidas entre ellos.

Como ejemplo, podemos mencionar la estrategia de la marca Gillette que ha preguntado: Como Superman hace la barba? La empresa inició la campaña el mismo período del estreno de la película Man of Steel (El hombre de Acero), propiciando a la audiencia una inmersión en el universo de este súper héroe en busca de la respuesta. Entre cómics, dibujos animados, wikis, películas, series, fórums de discusión online, videos autónomos y producidos por la marca etc, la audiencia (interna y externa) ha interaccionado con la marca, en forma de narrativa.

Es importante resaltar que la decisión de elección de los medios para la comunicación integrada debe tener en cuenta: el tipo de comunicación (organizacional, institucional, mercadológica, interna y administrativa), la audiencia que se quiere atingir, el tono del contenido/mensaje que será utilizado y la experiencia de envolvimiento o participación ofrecida. Así, es necesario, primero, conocer las especificidades y potencialidades de cada medio para, entonces, poder evaluarlo en lo que se refiere a los objetivos (macros y micros) de mercado y de comunicación de la marca.

Bautista (apud Pratten, 2011) propone la clasificación de los medios en una narrativa transmedia en dos formas de participación y dos formas de experiencia. Son ellas: participación pasiva y participación activa, experiencia individual y experiencia social. Con esta clasificación, la decisión de elección de los medios se torna más criteriosa y consciente a los objetivos de comunicación.

La narrativa transmedia permite que el mensaje sea apropiado por el individuo, siguiendo, interaccionando y haciendo conexiones entre los elementos de la narrativa a través de variados medios. Es a partir de la ruta seleccionada por el individuo al explorar el universo, que él construye el mensaje de forma única y no en una secuencia obligatoria impuesta por la marca.

Una gestión comunicacional, cuando bien hecha, utilizando la narrativa transmedia, propicia un universo inagotable a ser explorado en la relación mensaje-audiencia/marca-consumidos, pautados en macro y micro objetivos de mercado y comunicación.

\section{Una Estrategia y Experiencia Comunicacional}

La comunicación integrada, aliada a la narrativa transmedia, propicia una visión sistémica de la relación bidireccional marca-consumidor en todos los niveles e instancias. Al mismo tiempo en el que crea una sinergia entre las partes para que el mensaje de la marca sea visto como un sistema interconectado, en el que es posible conocer el impacto de cada acción en el todo.

Conforme Lupetti (2007, p. 15), "la comunicación integrada establece una política global, en función de la existencia de coherencia entre los programas establecidos en las comunicaciones institucional, administrativa, interna y mercadológica, además de evitar las sobre posiciones de tareas". De esta forma, la comunicación integrada puede ser el resultado de esfuerzos individuales, pues la imagen de la organización debe ser única y el proceso de toma de decisiones debe ser compartido por todos en las empresas.

En los últimos años, las empresas vienen observando la mudanza fundamental en el dominio de la comunicación con el uso de Internet y de las redes móviles como plataformas de comunicación digital. Ese tipo de comunicación está basada en redes horizontales de comunicación interactiva que, generalmente, son difíciles de controlar por parte de los gobiernos o de las empresas. De esta manera, se percibe que a través de Internet se tiene una estructura más abierta, libre y colaborativa.

Castells (2012) al hablar de las sociedades conectadas en red, presenta un análisis pionero sobre las características sociales innovadoras: conexión y comunicación horizontales; ocupación del espacio público urbano; creación de tiempo y de espacio propios; ausencia de líderes y de programas; aspecto al mismo tiempo local y global, tan pertinentes a nuestra discusión.

Por otro lado, Jenkins (2010) propone una ecología comunicativa que refleja el modo como nos comunicamos y nos relacionamos en la cultura contemporánea: en colectividad, en conexión, en colaboración y en participación, abriendo así espacio para una mirada más precisa sobre la cena mediática digital.

Al utilizar el término Spreadable media, en el sentido de medios pervasivos, Jenkins (2013) afirma:

Pervasividad se refiere a aquellos recursos técnicos que facilitan la circulación de determinados contenidos en detrimento de otros, a las estructuras económicas que sostienen o restringen esta circulación, a aquellos atributos de un contenido mediático que incitan la motivación de la 
comunidad a compartir, y a las redes sociales que conectan las personas a través del intercambio de bytes llenos de significado (JENKINS apud CORREAA, 2013, p.286).

El autor apunta que la cultura de la participación debe ser reconsiderada y reposicionada, integrando el concepto de reelaboración de mensajes y contenidos. Este tipo de participación puede generar nuevos formatos mediáticos en el contexto de una cultura conectada.

En este sentido, posicionamos la narrativa transmedia como el elemento que interconecta las partes del sistema comunicacional en un universo a ser explorado por todos los individuos consumidores de la marca, sean ellos gestores, funcionarios, subcontratados, clientes y potenciales clientes, renovando la marca continuamente.

Jenkins (2008, p. 138) afirma que "la comprensión obtenida por medio de diversos medios sostiene una profundidad de experiencia que motiva más consumo [...] Ofrecer nuevos niveles de revelación y experiencia nueva la marca y sostiene la fidelidad del consumidor". Por lo tanto, entendemos que las empresas pueden tener una mejor eficiencia en la gestión de los cuatro pilares de la comunicación organizacional para que haya una relación comunicacional continua y duradera entre marcas y consumidores, potencializando la efectividad de la compra

Como ya ha sido apuntado, el consumidor establece con la marca relaciones cognitivas emocionales y expresa diferentes sentimientos con relación a ella. Con una marca sólida, una organización expande su actuación en el mercado y combate más fuertemente la competencia y las adversidades del mercado.

\section{Consideraciones finales}

La narrativa transmedia, sea en el entretenimiento, en la educación o en la gestión comunicacional, es una estrategia de consumo de contenido y mensajes que busca atender las expectativas y demandas de una cultura participativa que se posiciona ora como audiencia y ora como productora. En esta integración de papeles queda evidente que los hábitos han cambiado, las experiencias se tornan más envolventes y ricas en interacción, mediadas, muchas veces por pantallas.

André Lemos resalta que la cibercultra se refiere a una forma sociocultural que modifica los hábitos sociales, prácticas de consumo cultura, ritmos de producción y distribución de la información, creando nuevas relaciones en el trabajo y en el ocio, nuevas formas de sociabilidad y de comunicación" (2010, p. 22).

Son estas nuevas relaciones de interacción y consumo que visualizamos, y que pasan a ser estimuladas y propiciadas con una actuación más próxima y envolvente entre el consumidor y la marca. Como ejemplo, algunas marcas crean sus propias redes sociales, pues entienden el mercado de consumo como un espacio abierto para el dialogo e intentan, de esta manera, establecer un diálogo efectivo con sus consumidores. La medida de este contacto en dos direcciones, entre el consumidor y la marca, viene pautando la toma de decisiones acerca de nuevos productos que serán lanzados y otras oportunidades de actuación de la marca.

Es importante considerar que, al trabajar con Comunicación Integrada, las herramientas presentadas tienen aplicaciones propias, con sus puntos fuertes y puntos débiles, como también, cuando utilizamos la Narrativa Transmedia, con su movimiento de contenido o mensaje en las varias plataformas de medios, es necesario que los discursos queden claros, y el enunciado en cada uno de los medios se resuelva por sí mismo, pero que tenga una línea narrativa que enlace estas distintas plataformas y posibilite una mejor comprensión que sea, a la vez, interesante. De alguna manera, recompensando los consumidores que se han dedicado y se han envuelto con la marca en sus diferentes momentos.

\section{Referencias Bibliográficas}

CASTELLS, Manuel. (2000): A sociedade em rede. Rio de Janeiro: Paz e Terra.

(2013): A era da intercomunicação. En Ministério da Cultura on-line [http://www2.cultura.gov.br/site/2006/08/18/a-era-da-intercomunicacao-por-manuel- castells, consultado el: 05/08/2013].

_. (2013): Redes de indignação e esperança. Movimentos sociais na era da internet. São Paulo: Editora Zahar.

CASTRO, Gisela. (2011): "Comunicação, consumo e capital humano: cultura digital e a mercantilização das subjetividades", En Freire Filho, João; Coelho, Maria das Graças Pinto, A promoção do capital humano: mídia, subjetividade e o novo espírito do capitalismo, Porto Alegre: Sulina.

_. (2009): "Nas tramas da rede". En: CAEPM (org.). Bravo mundo novo: as configurações da comunicação e do consumo. São Paulo: Alameda, pp. 43-62.

CORRÊA, Elizabeth Saad (2013): "Uma reconfiguração cultural possível e viável”. En Revista Matrizes Ano 7, n. 1 jan.jjun., p. 283-289. 
JENKINS, Henry. (2009): Cultura da Convergência, Rio de Janeiro: Aleph.

JENKINS, Henry et al. (2013): T is for Transmedia: Learning through transmedia play. Los Angeles and New York: USC Annenberg Innovation Lab and The Joan Ganz Cooney Center at Sesame Workshop. [http://www.annenberglab.com/viewresearch/46, consultado el: 10/05/2013].

KUNSCH, Margarida (2003): Planejamento de relações públicas na comunicação integrada. São Paulo: Summus Editorial.

LEMOS André. y LÉVY, Pierre. (2010): O futuro da internet: em direção a uma ciberdemocracia planetária, São Paulo: Editora Paulus.

LUPETTI, Marcélia. (2007): Gestão estratégica da comunicação mercadológica. São Paulo: Thompson Learning.

PRATTEN, Robert (2011): Getting started in transmedia storytelling: a practical guide for beginners. USA: Robert Pratten. 